\title{
Actionable Real-World Evidence to Improve Health Outcomes and Reduce Medical Spending Among Risk-Stratified Patients with Diabetes
}

\author{
Elizabeth M. Garry, PhD, MPH; Sebastian Schneeweiss, MD, ScD; Sara Eapen, PhD; \\ Natalia Petruski-lvleva, PhD, MS; Elizabeth Cheever, MS; William Murk, PhD, MPH; Aditya Rajan, MPH; \\ Jeremy A. Rassen, ScD; David Gambino, MS; Hannah Jang, PharmD; \\ Elizabeth Rubin, MPA; and Saira Jan, MS, PharmD
}

\section{ABSTRACT}

BACKGROUND: Type 2 diabetes mellitus (T2DM) is a chronic condition with a high economic burden as well as drug treatments that have not all demonstrated effects on longevity. Managed care organizations want to improve health outcomes in these complex patients but lack actionable evidence to make informed decisions on which therapies are most effective among their members and may also control total health care spending.

OBJECTIVE: To produce actionable evidence by identifying antidiabetic treatments that are effective and may reduce total cost of care in various risk groups of patients with T2DM, using insurance claims data that includes medical claims and pharmacy dispensing data among members of Horizon Blue Cross Blue Shield of New Jersey with T2DM.

METHODS: We identified patients with T2DM in longitudinal claims data from Horizon between 2014 and 2017 with demographic and enrollment information, inpatient and outpatient diagnoses and procedures, and pharmacy dispensing. Outcomes included myocardial infarction, heart failure (HF), stroke, percutaneous revascularization, health care services utilization, and plan costs (i.e., medical, pharmacy, and total cost of care). After propensity score decile adjustment on over 20 covariates, we evaluated the effectiveness and safety of second-line antidiabetic treatment that included sodium-glucose co-transporter-2 (SGLT-2) inhibitors, sulfonylureas (SUs), dipeptidyl peptidase-4 (DPP-4) inhibitors, and glucagon-like peptide-1 (GLP-1) receptor agonists.

RESULTS: Among 115,308 members with T2DM, the most common comorbidities were cardiovascular risk factors, including hyperlipidemia (56\%), hypertension (50\%), and existing cardiovascular disease (CVD; 55\%). Among members receiving dual antidiabetic treatment $(n=20,204)$, the most prevalent treatments were metformin plus the following second-line medications: SUs (42\%), DPP-4 inhibitors (29\%), SGLT-2 inhibitors (10\%), or GLP-1 receptor agonists (3\%). Approximately $20 \%$ of members accounted for $79 \%$ of total cost of care, with an average of $\$ 9,605$ per member per year (PMPY). Compared with SU initiation and after propensity score decile adjustment, new users of SGLT-2 inhibitors had a reduced risk for $\mathrm{HF}$ hospitalization ( $\mathrm{HR}=0.35,95 \% \mathrm{Cl}=0.13-0.89$ ), hypoglycemia, albuminuria, microvascular disease, and metabolic failure. Among SGLT-2 inhibitor initiators with established CVD, the savings in total cost of care compared with SU initiators was $\$ 5,520$ per member over an average treatment duration of 6 months and an approximate savings of $\$ 11,000$ PMPY if patients persisted on treatment for 12 months.
CONCLUSIONS: In the Horizon membership, we confirmed that SGLT-2 inhibitors reduce HF hospitalizations, resulting in reduced medical spending and savings in total cost of care. Regulatory-grade analytics of local data provided the confidence to encourage increased SGLT-2 inhibitor use to produce better outcomes and save total cost of care despite higher pharmacy spending.

J Manag Care Spec Pharm. 2019;25(12):1442-52

Copyright $\odot 2019$, Academy of Managed Care Pharmacy. All rights reserved.

\section{What is already known about this subject}

Prevalence of type 2 diabetes, with its high economic burden and known treatment challenges, continues to rise.

Managed care organizations responsible for improving health outcomes in their patient populations are faced with escalating health care costs with cost drivers varying across different payer types, provider organization, geography, and therapeutic areas. Newer antidiabetic treatments have been approved to control glycemic levels and improve cardiovascular outcomes, but payers are wary to cover expensive drugs and often lack confidence in real-world evidence to inform their decision making due to a lack of scientific validation.

\section{What this study adds}

This study illustrates one payer's solution of using regulatorygrade rapid analytics of its own plan-specific, real-world data to inform treatment care pathways and cost containment strategies in the payer's targeted populations without solely relying on evidence from clinical trial data or national databases, which may not adequately represent its own members with type 2 diabetes. By risk stratifying the plan's type 2 diabetes population, it was found that the more expensive second-line antidiabetic treatment class of sodium-glucose co-transporter-2 inhibitors may produce significant medical cost savings for the payer's members at high risk for cardiovascular disease rehospitalization.

Analysis in this study confirmed clinical trial evidence and produced plan-specific evidence, highlighting the importance of generating real-world evidence in a payer's own database that can be used for value-based contracting. 


\section{Actionable Real-World Evidence to Improve Health Outcomes and Reduce Medical Spending Among Risk-Stratified Patients with Diabetes}

$\mathrm{T}$ he majority of health care costs are attributable to a disproportionately small number of patients. ${ }^{1}$ In the United States, $5 \%$ of the population is responsible for over half of the health care spending. ${ }^{2}$ Compounding the problem, high-cost patients tend to be persistent spenders in subsequent years. ${ }^{3}$ The typical high-cost patient is complex and often has multiple chronic conditions, such as diabetes and ischemic heart disease, that impair different body systems. ${ }^{4,5}$

Diabetes is a chronic condition with a high economic burden that is expected to double over the next 15 years. ${ }^{6}$ In the United States, over 30 million people have type 2 diabetes mellitus (T2DM), representing an estimated $\$ 245$ billion in health care costs. ${ }^{7}$ Factors associated with high costs in diabetes care are rooted in insufficient glucose control and existing comorbidities, including renal impairment, obesity, hyperlipidemia, hypertension, and coronary heart disease. ${ }^{8}$ Furthermore, new antidiabetic treatments have emerged, demonstrating efficacy in glycemic control and cardiovascular risk reduction in clinical trials, but high cost and slow knowledge dissemination of the most effective treatment in real-world populations slows adoption of new treatments, including those with incremental benefits. Given rising health care spending, managed care organizations are interested in better understanding these complex high-cost patients to implement effective cost-containment strategies and targeted interventions. While real-world data (RWD) is essential for biopharmaceutical manufacturers and regulatory agencies, ${ }^{9,10}$ payers cite substantial barriers to using RWD for decision making, including lack of transparency, lack of randomization, complex methodologies that are difficult to understand by decision makers, and timeliness of real-world evidence (RWE). ${ }^{11}$

Using RWD from T2DM members of Horizon Blue Cross Blue Shield of New Jersey (BCBSNJ), we sought to evaluate the high-cost patients with T2DM to inform care management and effective cost-containment strategies. Our analytic approach consisted of 3 steps: (1) characterize members with T2DM with respect to their demographics, medical history, treatment patterns, resource utilization, and costs to identify drivers of expenditures; (2) identify the high-cost, high-risk population that would benefit most from targeted interventions; and (3) identify classes of drugs that affected cost drivers in high-risk populations. The analyses were designed to inform value-based contracting and targeted prescribing interventions to improve care while materializing economic benefits.

\section{Methods}

We used a population-based cohort design to evaluate patients diagnosed with T2DM in longitudinal patient-level claims data from Horizon BCBSNJ, a regional managed care provider serving the U.S. state of New Jersey.

\section{Data Source}

Horizon BCBSNJ provided administrative claims data between January 1, 2014, and August 31, 2017, which contained demographic and enrollment information and data on inpatient and outpatient facility visit diagnoses and procedures as well as pharmacy dispensing.

\section{Study Population}

Descriptive Cohort. The overall study population comprised all patients aged 18 years or older with at least 1 diabetes diagnosis (International Classification of Diseases, Ninth/Tenth Revision, Clinical Modification codes: ICD-9-CM codes 250.X0, 250.X2, and 790.2; ICD-10-CM codes E11 and R73.0) or at least 1 claim for diabetes-related drugs (General Product Identifier code 27 for antidiabetics or 9729 for diabetic supplies) between January 1, 2014, and August 31, 2017. Patients must have filled at least 1 prescription claim to be included in the analysis. The cohort entry date was defined as the first observed date of diagnosis or first antidiabetic treatment dispensing.

Comparative Cohorts. We created 3 cohorts to conduct pairwise comparisons between sodium-glucose co-transporter-2 (SGLT-2) inhibitors and other second-line treatment groups. The comparative cohorts comprised new users of SGLT-2 inhibitors, dipeptidyl peptidase-4 (DPP-4) inhibitors, glucagon-like peptide-1 (GLP-1) receptor agonists, or sulfonylureas (SUs) between January 1, 2015, and December 31, 2016, with continuous enrollment and no use of basal insulin during baseline (180 days before cohort entry). New use was defined as requiring a washout period during baseline. Members were included in each comparison based on their first qualifying treatment arm only, with the cohort entry date defined according to the first qualifying dispensing of the drug of interest.

\section{Medication Exposure}

First-line treatment was defined as a dispensing of metformin. New use of second-line antidiabetic treatment of interest alone or in combination with metformin included SGLT-2 inhibitors (canagliflozin, dapagliflozin, and empagliflozin); DPP-4 inhibitors (alogliptin, linagliptin, saxagliptin, and sitagliptin); GLP-1 receptor agonists (exenatide, dulaglutide, albiglutide, liraglutide, and lixisenatides); or second-generation SUs (glimepiride, glipizide, and glyburide).

\section{Outcomes}

Outcomes comprised the following: (a) health outcomes, including urinary tract infections (UTIs); microvascular complications (retinopathy, nephropathy, and neuropathy); myocardial infarction (MI); stroke; angina; heart failure (HF) hospitalization; diabetic ketoacidosis; hypoglycemia; albuminuria; kidney failure; metabolic failure; and percutaneous revascularization as defined by ICD-9/10-CM codes (Appendix A, available in 


\section{Actionable Real-World Evidence to Improve Health Outcomes and Reduce Medical Spending Among Risk-Stratified Patients with Diabetes}

online article); (b) health care services utilization, including office visits, hospitalizations, and prescription drug dispensings; and (c) health care cost, consisting of any medical, hospital, pharmacy, and total cost of care. Costs included payer and patient expenditures and were expressed as per member per year (PMPY). These costs were calculated as the total average paid divided by the average treatment duration, multiplied by 365 days. For the analyses of cost percentiles, costs were calculated for each patient as the total paid divided by treatment duration, multiplied by 365 days.

\section{Patient Characteristics}

More than 20 relevant patient characteristics were reported during baseline, which included demographic information (age, gender, and insurance type) and cardiovascular-related comorbidities (hypertension, hyperlipidemia, obesity, smoking, and coronary artery bypass graph). Cardiovascular disease (CVD) included MI, HF, atrial fibrillation, angina, stroke, peripheral vascular disease, coronary artery disease, or peripheral arterial disease. Other comorbidities included chronic obstructive pulmonary disease; renal disease; pain disorders (migraine, rheumatoid arthritis, and chronic, back, joint, or chest pain); sleep disorders (chronic fatigue syndrome, anemia, sleep apnea, and malaise); and depression. We also included use of antihypertensives, aspirin, beta blockers, statins, and other diabetic medications. Cost analyses additionally adjusted for total costs for pharmacy, inpatient medical, outpatient medical, and emergency room claims during baseline.

\section{Cardiovascular Risk Groups}

We defined 3 patient subgroups by high-risk CVD status (i.e., patients with CVD-related hospitalization any time before cohort entry); medium-risk status (i.e., patients with previous CVD-related office visits but no hospitalization); and no recorded risk.

\section{Statistical Analysis}

Descriptive analyses were performed to characterize the patient population, comorbidities, treatment patterns, and resource utilization. Adjusted deciles of propensity score and Cox proportional hazards regression models were used for the pairwise comparisons between SGLT-2 inhibitors and SUs and between SGLT-2 inhibitors and DPP-4 inhibitors. Comparisons between SGLT-2 inhibitors and GLP-1 receptor agonists were considered but not executed because of the small number of patients exposed. ${ }^{12}$ Patient follow-up for outcome ascertainment started the day after cohort entry and was censored at the occurrence of an outcome, disenrollment, end of study period, treatment discontinuation, or initiation of another second-line antidiabetic treatment. Cost outcomes were modeled utilizing a generalized linear model that used a linear link function to estimate differences in mean cost and a negative binomial error distribution to accommodate right-skewed distributions of cost.

\section{TABLE 1 Patient Characteristics}

\begin{tabular}{l|rr}
\hline Characteristic & $n(\%)$ \\
\hline $\begin{array}{l}\text { Total population of patients with T2DM or medication use } \\
\text { Demographics }\end{array}$ & $56(48,308$ & $(100.0)$ \\
\hline Age, median (IQR) in years & & \\
\hline Gender & 53,561 & $(46.5)$ \\
\hline Female & 61,747 & $(53.5)$ \\
\hline$\quad$ Male & & \\
\hline Clinical characteristics & 64,875 & $(56.3)$ \\
\hline Hyperlipidemia & 63,563 & $(55.1)$ \\
\hline Cardiovascular diseasea & 57,830 & $(50.2)$ \\
\hline Hypertension & 51,069 & $(44.3)$ \\
\hline Pain disorders & 30,661 & $(26.6)$ \\
\hline Fatigue and sleep-related disorders & 23,410 & $(20.3)$ \\
\hline Obesity & 7,399 & $(6.4)$ \\
\hline Liver disease, cirrhosis, and other liver conditions & 7,080 & $(6.1)$ \\
\hline Chronic obstructive pulmonary disease & 9,993 & $(8.7)$ \\
\hline Infectious complications & 6,511 & $(5.6)$ \\
\hline Depression & 6,121 & $(5.3)$ \\
\hline Any cancer & 5,886 & $(5.1)$ \\
\hline Stroke & &
\end{tabular}

ancludes myocardial infarction, heart failure, atrial fibrillation, angina, stroke, peripheral vascular disease, coronary artery disease, and peripheral arterial disease.

$I Q R=$ interquartile range; $T 2 D M=$ type 2 diabetes mellitus.

All analyses were performed using Aetion Evidence Platform, version 3.0, which has previously been validated for a range of studies and for predicting clinical trial findings. ${ }^{13,14}$ The platform was available to the decision makers to view and reproduce findings and conduct sensitivity analyses with changed assumptions in real time.

\section{Results}

\section{Population and Care Pathways}

Among the 248,064 members with T2DM enrolled in Horizon BCBSNJ, 115,308 patients met the eligibility criteria. The median age was 56 years (interquartile range: 48-63), and $53.5 \%$ were male (Table 1). The most common comorbidities were CVD (55.1\%) or CVD risk factors, including hyperlipidemia (56.3\%), hypertension (50.2\%), and obesity (20.3\%). Other common comorbidities included pain (44.3\%) and fatigue or sleep-related disorders (26.6\%). Depression was recorded among $5.6 \%$ of patients. Multimorbidity (2 or more chronic conditions) appeared common in this patient population.

Approximately $34.4 \%(n=39,657), 17.5 \%(n=20,204)$, and $14.3 \%(n=16,450)$ of members received metformin only, dual therapy, or other combination therapy, respectively (Appendix B, available in online article). Among members receiving dual therapy, the most prevalent treatment combined with metformin was SUs (41.5\%); $29.0 \%$ used DPP-4 inhibitors; $10.0 \%$ used SGLT-2 inhibitors; and less than 5\% used GLP-1 receptor agonists or other antidiabetic treatment. The average treatment 


\section{Actionable Real-World Evidence to Improve Health Outcomes and Reduce Medical Spending Among Risk-Stratified Patients with Diabetes}

\section{FIGURE 1 Resource Utilization and Percentage of Costs by PMPY Cost Distribution}

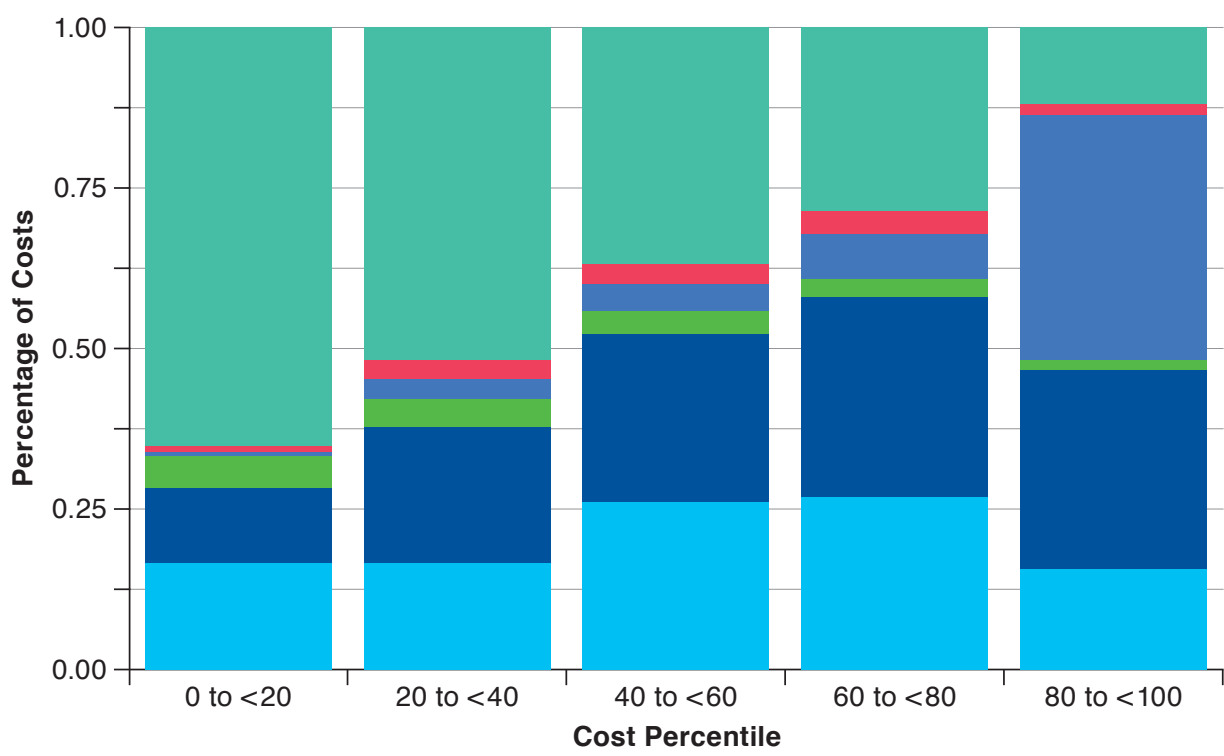

\section{Setting}

Doctor's Office

Emergency Room

Inpatient

Laboratory

- Outpatient

Pharmacy

Note: Cost percentages were calculated out of the PMPY total costs within each percentile category based on all patients with at least 1 ICD code for T2DM (N = 248,064). Of those, 115,308 had at least 1 drug dispensing during baseline and were further analyzed.

ICD = International Classification of Diseases; PMPY = per member per year; T2DM = type 2 diabetes mellitus.

persistence was 6 months since initiation. A total of 38,997 patients (34\%) had no diabetes-related pharmacy claims. The percentage of patients using statins during baseline for each second-line therapy group evaluated was $42 \%, 46 \%$, and $52 \%$ for SU, DPP-4 inhibitor, and SGLT-2 inhibitor users, respectively.

\section{Resource Utilization}

Overall, 20\% of members accounted for $79 \%$ of costs paid, with an average of $\$ 9,605$ PMPY. Figure 1 shows the distribution of total costs among all T2DM beneficiaries. Among patients in the top 20th percentile, members had a higher prevalence of comorbidities than their lower-cost counterparts, including cardiovascular risk factors ( $80.9 \%$ vs. $66.3 \%$ ), pain disorders ( $77.1 \%$ vs. $53.6 \%$ ), sleep disorders ( $52.0 \%$ vs. $31.3 \%$ ), obesity ( $37.0 \%$ vs. $23.1 \%$ ), and depression ( $14.3 \%$ vs. $6.5 \%$ ).

Hospitalizations were driving expenditures among the high-cost patients, for which inpatient stays accounted for $38.3 \%$ of the total costs, followed by outpatient facility visit costs $(31.2 \%)$, pharmacy dispensing (15.5\%), doctor's office visits (12.4\%), and emergency room visits (1.5\%). Interestingly, inpatient costs ranged from $0.67 \%$ to $7.4 \%$ for the remaining members in the lower $80 \%$ quintiles (Figure 1).

\section{Health Outcomes}

The hazard ratio (HR) estimates comparing SGLT-2 inhibitors to SUs and SGLT-2 inhibitors to DPP-4 inhibitors for clinical outcomes during follow-up that adjust for propensity score deciles are summarized in Figure 2. Compared with SU initiators, SGLT-2 inhibitor initiators had a reduced risk for hypoglycemia $(\mathrm{HR}=0.19,95 \%$ confidence interval $[\mathrm{CI}]=0.08$ 0.50); $\mathrm{HF}$ hospitalization ( $\mathrm{HR}=0.35,95 \% \mathrm{CI}=0.13-0.89$ ); albuminuria $(\mathrm{HR}=0.42,95 \% \mathrm{CI}=0.22-0.79)$; microvascular disease $(\mathrm{HR}=0.61,95 \% \mathrm{CI}=0.50-0.74)$; and metabolic failure $(\mathrm{HR}=0.72,95 \% \mathrm{CI}=0.56-0.92)$. The results for the association of SGLT-2 inhibitors and other clinical outcomes, including hospitalization for MI or stroke, kidney failure, and hospitalization for composite CVD events (MI, stroke, angina, and revascularization) were suggestive of a reduced risk compared with SU use. There appeared to be no statistically significant association between SGLT-2 inhibitors and UTIs.

Compared with DPP-4 inhibitor initiation, SGLT-2 inhibitor initiation was associated with a decreased risk for hypoglycemia $(\mathrm{HR}=0.28,95 \% \mathrm{CI}=0.10-0.75)$; albuminuria $(\mathrm{HR}=0.48$, $95 \% \mathrm{CI}=0.24-0.94)$; and microvascular disease ( $\mathrm{HR}=0.74$, $95 \% \mathrm{CI}=0.60-0.92)$. We observed a trend towards risk reduction for metabolic failure, hospitalization for HF, and hospitalization for MI or stroke.

\section{Cost Outcomes}

Among T2DM patients initiating SGLT-2 inhibitors $(n=3,626)$ or SUs $(n=6,709)$ as second-line treatment with an average 6-month treatment duration, SGLT-2 inhibitor initiation was associated with an average increased pharmacy cost of $\$ 1,527$ per patient $(95 \% \mathrm{CI}=\$ 1,244-\$ 1,809)$. Figure 3 shows the mean 


\section{Actionable Real-World Evidence to Improve Health Outcomes and Reduce \\ Medical Spending Among Risk-Stratified Patients with Diabetes}

\section{FIGURE 2 HRs with 95\% Cls for SGLT-2 Inhibitors Versus SUs and DPP-4 Inhibitors}

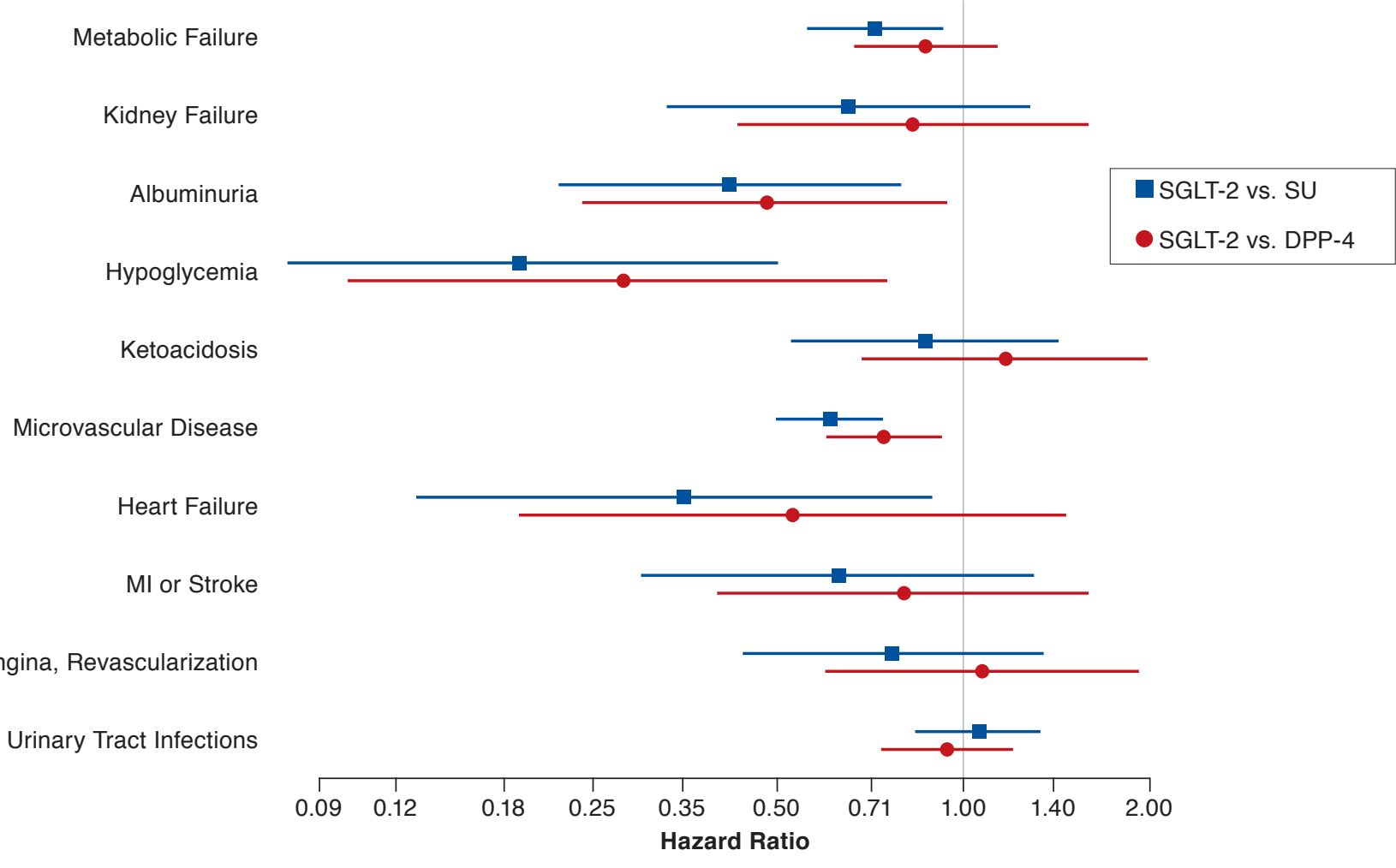

Note: More than 20 risk factors were adjusted for by a propensity score decile model. Estimated among patients with no history of the event of interest in the baseline

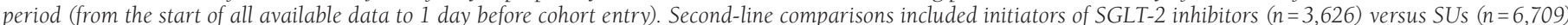

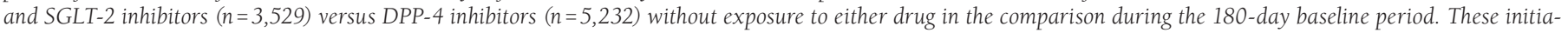
tors were aged at least 18 years, had no previous exposure to basal insulin, and had at least 1 medical claim in order to identify medical benefit. Second-line treatment with GLP-1 receptor agonists was considered but excluded due to small sample size.

$C I=$ confidence interval; DPP-4 = dipeptidyl peptidase-4; GLP-1 = glucagon-like peptide-1; HR=hazard ratio; MI=myocardial infarction; SGLT-2 = sodium-glucose co-transporter-2; SU = sulfonylurea.

cost differences per member stratified by CVD risk. In the CVD high-risk subgroup, the mean difference in total cost of care per patient between SGLT-2 inhibitor initiators and SU initiators was $-\$ 5,520$, leading to an estimated savings of about $\$ 11,000$ PMPY, assuming persistent use for a full year. The difference was primarily attributed to reduced total medical cost (difference: $-\$ 6,138$ [95\% CI =-\$13,610-\$1,334]) compared with SU initiators. This in turn was mostly driven by inpatient cost savings of $-\$ 4,643(95 \% \mathrm{CI}=-\$ 11,091-\$ 1,805)$ versus outpatient savings $-\$ 648(95 \% \mathrm{CI}=-\$ 2,546-\$ 1,251)$, respectively.

In comparing initiators of SGLT-2 inhibitors with initiators of DPP-4 inhibitors among patients in the CVD high-risk subgroup, the total mean cost difference per patient was $-\$ 3,419$ (95\% CI $=-\$ 11,264-\$ 4,426)$. Compared with DPP-4 inhibitors, there appeared to be a decreased in cost per patients initiating SGLT-2 inhibitors for pharmacy costs and medical costs; however, the results were not statistically significant.
Lower CVD-risk subgroups showed no clear patterns in cost savings for SGLT-2 inhibitor users.

\section{Discussion}

Our analytic approach resulted in the following 5 key findings, some of which confirmed previous known evidence, while other plan-specific results led to recommendations for targeted care delivery.

1. About $79 \%$ of total cost of care for patients with T2DM was attributable to $20 \%$ of members trackable in the health plan's data.

2. Among high-cost patients, drivers of costs included a high prevalence of CVD comorbidities and hospitalizations, factors placing patients at high-risk for a subsequent CVDrelated event.

3. In patients at high risk for CVD, the use of SGLT-2 inhibitors compared with SUs or DPP-4 inhibitors could result in 


\section{FIGURE 3 Mean Cost Difference Per Member Stratified by Cardiovascular Risk}

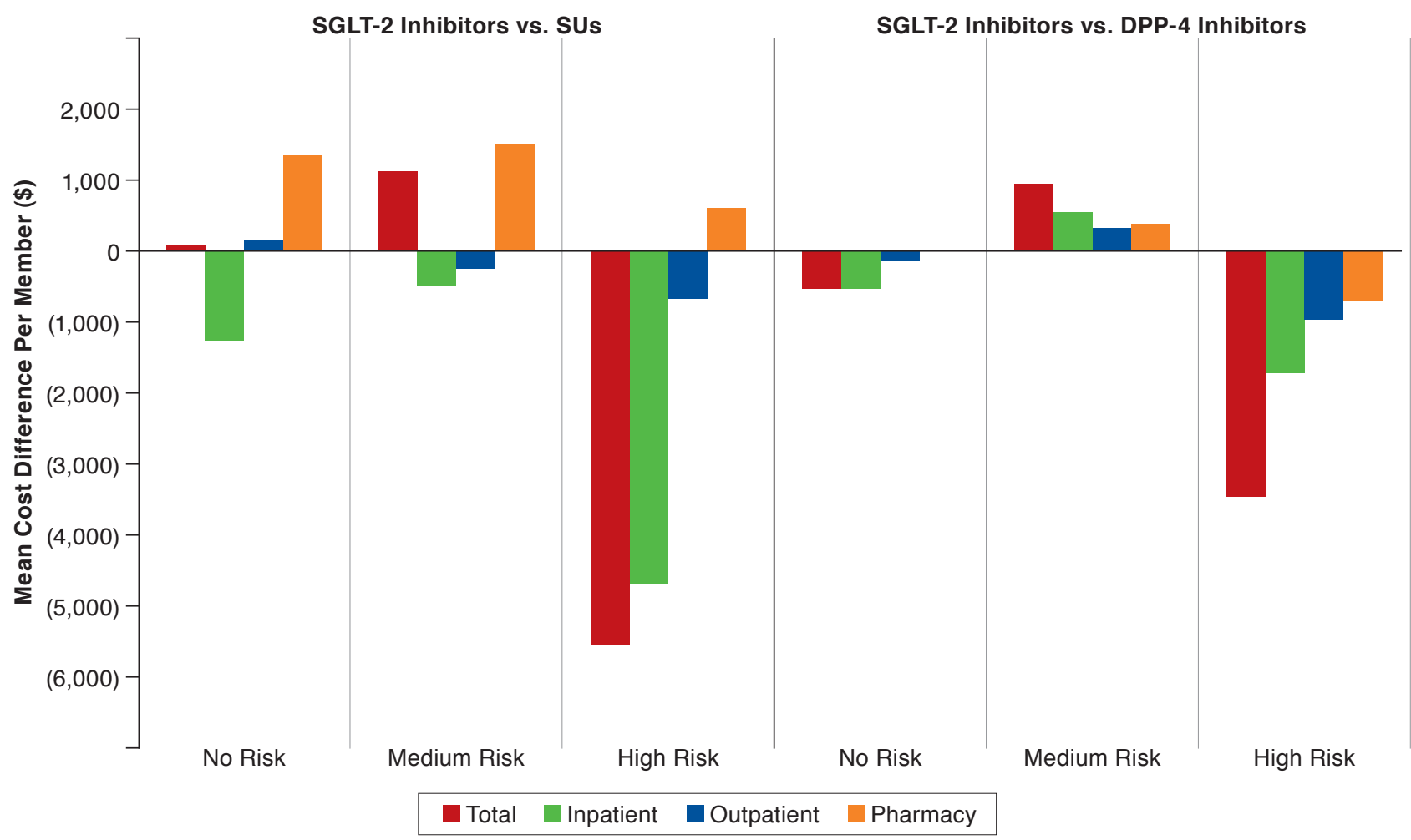

Note: Second-line comparisons included initiators of SGLT-2 inhibitors $(n=3,626)$ versus SUs $(n=6,709)$ and SGLT-2 inhibitors $(n=3,529)$ versus DPP-4 inhibitors $(n=5,232)$ without exposure to either drug in the comparison during the 180-day baseline period. These initiators were aged at least 18 years, had no previous exposure to basal insulin, and had at least 1 medical claim in order to identify medical benefit. Second-line treatment with GLP-1 receptor agonists was additionally considered but omitted from the analysis due to small sample size.

DPP-4 = dipeptidyl peptidase-4; GLP-1 = glucagon-like peptide-1; SGLT-2 = sodium-glucose co-transporter-2; SU = sulfonylurea.

meaningfully better health outcomes and substantial savings in medical costs, despite initial higher drug cost.

4. Second-line antidiabetic treatment was suboptimal in terms of the substantial underuse of SGLT-2 inhibitors and an average 6-month treatment duration.

5. Using the payer's own data, we identified an opportunity for the optimization of care currently provided to patients with T2DM by prioritization of SGLT-2 inhibitor use and incentivizing persistence, especially among patients at high risk for CVD-related events.

Using a health plan's RWD claims, we segmented the high-cost patients in order to find drivers of costs and identified a class of drugs that has the potential to lower overall medical costs. We found that the more expensive second-line antidiabetic treatment class of SGLT-2 inhibitors lowered the rate of HF hospitalizations among Horizon BCBSNJ members with T2DM who were at risk for costly CVD rehospitalization. Substantial savings in medical cost could be realized by increasing the use of SGLT-2 inhibitors among patients at high risk for CVD events, whether by increasing the SGLT-2 inhibitor uptake among eligible members and/or improvement in persistence to ensure cardio-protective effects. For example, among the approximately 2,000 enrolled members who are at high risk for CVD-related hospitalization, switching $75 \%$ of the members' dual therapy to SGLT-2 inhibitors and simultaneously improving persistence by 2 months would yield approximately $\$ 10.9$ million annual medical cost savings. Nonadherence to T2DM medication is well documented, ${ }^{15-17}$ and efforts are underway to examine interventions to improve antidiabetic treatment adherence and persistence. ${ }^{18,19}$

This analysis adds to the growing literature examining high-cost patients across different therapeutic areas and payer types..$^{20}$ In general, high-cost patients are characterized with a higher burden of disease, often with chronic illness and multimorbidity. High-cost risk factors include older age, lifestyle (e.g., obesity and smoking), clinical characteristics, and payer type. Several studies have evaluated preventable costs, such 


\section{Actionable Real-World Evidence to Improve Health Outcomes and Reduce Medical Spending Among Risk-Stratified Patients with Diabetes}

as avoidable hospitalizations, among these high-cost patients. Niefeld et al. (2003) evaluated Medicare members with T2DM who were aged 65 years or older and found that CVD-related comorbidities (e.g., congestive HF, cardiomyopathy, coronary atherosclerosis, hypertension, and cardiac dysrhythmias) and non-CVD comorbidities (e.g., chronic obstructive pulmonary disease and depression) increased the odds of preventable hospitalizations. Patients with T2DM along with congestive HF were more than 3 times as likely to experience a preventable hospitalization than those without. ${ }^{21}$ The association between multimorbidity and preventable hospitalizations may be due to suboptimal adherence of complex treatment regimens burdened by cumulative treatment recommendations, polypharmacy, and competing treatments by multiple physicians. ${ }^{22}$

Previous research also suggests that regional differences affect preventable costs per capita, with an association between higher availability of primary and specialty physicians within different hospital referral regions and higher preventable costs per capita. ${ }^{23}$ The complexity and diversity of a high-cost population requires that individual plans fully understand their specific high-cost populations and tailor interventions accordingly. Solutions to lowering costs are not "one size fits all"; segmentation analysis allows health plans to identify the subset of patients that would optimally benefit from targeted policies and interventions. ${ }^{24}$

The innovative application of using RWD to conduct near real-time comparative effectiveness analyses can help managed care organizations identify their unique high-cost patients and to address their unmet needs. In the case of this study, our findings enabled the payer to develop targeted pharmacy case management solutions and data-driven interventions, including health care system education and targeted patient outreach programs to improve persistence and understand treatment barriers among high-risk members, as well as engage in outcomes-based contracting with manufacturers.

\section{Strengths and Limitations}

Our case study has several notable strengths. First, regulatorygrade, rapid analytic technology enabled the analyses to be conducted in a timely (6 weeks), transparent, reproducible, and scientifically validated manner. The core analytic technology we employed is used by various regulatory pilot programs that evaluate RWE. ${ }^{25,26}$ The analytic platform generated auditable documentation on key assumptions and decisions as recommended by standard reporting guidelines. ${ }^{27-29}$

Second, our findings were consistent with published randomized controlled trials (RCT), despite different study populations in the international cardiovascular outcomes trials and our real-world analysis on local data. ${ }^{30-32}$ Our findings were also consistent with the glucocentric to cardiocentric treatment paradigm shift reflected in the recent 2019 American Diabetes Association clinical guidelines. ${ }^{33}$ Nonrandomized studies are considered as valid as RCTs for certain clinical questions. ${ }^{34}$ While RCT data and national claims data analyses had provided consistent evidence of reduced HF hospitalizations with SGLT-2 inhibitor use, our findings provided assurances to Horizon BCBSNJ that health outcomes and subsequent savings would translate to its own population. ${ }^{35,36}$ In addition, we used robust statistical methods, such as risk-adjusted propensity score analyses to adjust for patient characteristics in the treatment and comparison groups. Finally, by engaging a third-party, Horizon BCBSNJ avoided using internal resources to conduct analyses on their own RWD, another known barrier of RWE use. ${ }^{11}$

This study also has some limitations to consider. We used regional data representing commercially insured, continuously enrolled members, so findings may not be generalizable to Medicare or Medicaid populations. Reassuringly, our results were consistent with published literature using nationally representative data. ${ }^{35}$ Our study has known limitations common to administrative claims data structured for transactional payment and not research, including lack of clinical documentation, misdiagnosis, and miscoding. ${ }^{37}$ However, we were able to reproduce trial findings and demonstrate robust results.

While we adjusted for over 20 patient characteristics, we cannot fully rule out the presence of unmeasured confounders. Horizon BCBSNJ's commercial market segment requires no prior authorization for SGLT-2 inhibitors; empagliflozin and canagliflozin have preferred formulary status for making socioeconomic status an unlikely cause for bias. Recent evidence also suggests cardio-protective effects with GLP-1 receptor agonists. ${ }^{38}$ However, our limited study size prevented the comparison between SGLT-2 inhibitors with this class of antidiabetic treatment, since only 3\% of T2DM members were prescribed GLP-1 receptor agonists, representing a negligible pharmacy share.

\section{Conclusions}

It is well established that diabetic populations are complex, with a high prevalence of coexisting comorbidities and costly treatment pathways. Risk stratifying a private insurance plan's T2DM population yielded nonintuitive results: The more expensive second-line antidiabetic treatments may produce significant cost savings and improved patient health outcomes for the current 2,000 enrolled members at high risk for a severe cardiovascular event. Regulatory-grade and rapid analytics of RWD enabled the payers to develop targeted pharmacy case management solutions and data-driven interventions, including health care systems education and patient outreach programs to improve medication persistence and understand treatment barriers among high risk CVD members as well as engage in outcomes-based contracting with manufacturers. 


\section{Actionable Real-World Evidence to Improve Health Outcomes and Reduce Medical Spending Among Risk-Stratified Patients with Diabetes}

\section{Authors}

ELIZABETH M. GARRY, PhD, MPH; SARA EAPEN, PhD; NATALIA PETRUSKI-IVLEVA, PhD; ELIZABETH CHEEVER, MS; WILLIAM MURK, PhD, MPH; ADITYA RAJAN, MPH; and JEREMY A. RASSEN, ScD, Aetion, New York, New York. SEBASTIAN SCHNEEWEISS, MD, ScD, Division of Pharmacoepidemiology and Pharmacoeconomics, Department of Medicine, Brigham \& Woman's Hospital, Boston, and Harvard Medical School, Boston, Massachusetts. DAVID GAMBINO, MS; HANNAH JANG, PharmD; and ELIZABETH RUBIN, MPA, Department of Pharmacy Practice and Administration, Pharmacy Strategy and Clinical Integration, Horizon Blue Cross Blue Shield of New Jersey, Newark. SAIRA JAN, MS, PharmD, Department of Pharmacy Practice and Administration, Pharmacy Strategy and Clinical Integration, Horizon Blue Cross Blue Shield of New Jersey, Newark, and Rutgers State University of New Jersey School of Pharmacy, Piscataway.

AUTHOR CORRESPONDENCE: Elizabeth M. Garry, PhD, MPH, Aetion, 50 Congress St., Boston, MA 02109. Tel.: 844.823.8466; E-mail: liz.garry@aetion.com.

\section{DISCLOSURES}

This research did not receive outside funding; however, Aetion has since begun a contractual relationship with Horizon Blue Cross Blue Shield of New Jersey.

Garry, Petruski-Ivleva, Cheever, and Rajan are employees of and have stock options in Aetion, a company that makes software for the analysis of real-world data. Eapen was an employee of Aetion during the implementation of this study. Rassen is an employee of and has ownership interest in Aetion. Murk is a consultant to Aetion of which he owns equity. Schneeweiss is a consultant to WHISCON and to Aetion, of which he also owns equity. He is the principal investigator of investigator-initiated grants to the Brigham and Women's Hospital from Bayer, Genentech, Boehringer Ingelheim, and Vertex. Gambino is an employee and officer at Horizon Blue Cross and Blue Shield of New Jersey. He was recently appointed to a board observer position at Aetion, as Horizon has small equity interest in Aetion. Jan is an employee of Rutgers State University and Horizon Blue Cross Blue Shield of New Jersey and has no conflict of interest or association with Aetion or any pharmaceutical company. Jang and Rubin are employees of Horizon Blue Cross and Blue Shield of New Jersey and have no conflict of interest or association with Aetion.

This work was presented as a poster at AMCP Nexus 2018, October 22-25, 2018, in Orlando, FL; as part of a continuing education session at the AMCP Managed Care \& Specialty Pharmacy 2019 Annual Meeting in San Diego, CA, March 25-28, 2019; as invited podium presenter at the Blue Cross Blue Shield 2019 National Summit conference in Grapevine, TX, April 29-May 2, 2019; and was accepted for a podium presentation at the International Society for Pharmacoeconomics and Outcomes Research (ISPOR) 2019 annual conference in New Orleans, LA, May 18-22, 2019, where it won an award for Best Podium Presentation.

\section{ACKNOWLEDGMENTS}

The authors acknowledge Pattra Mattox for providing medical writing support and Mohit Misra for providing clinical expertise.

\section{REFERENCES}

1. McWilliams JM, Schwartz AL. Focusing on high-cost patients-the key to addressing high costs? N Engl J Med. 2017;376(9):807-09.
2. Cohen S, Uberoi N. Differentials in the concentration in the level of health expenditures across population subgroups in the U.S., 2010. Statistical Brief \#421. August 2013. Agency for Healthcare Research and Quality. Rockford, MD. Available at: https://meps.ahrq.gov/data_files/publications/st421/stat421.shtml. Accessed November 12, 2019.

3. Coughlin TA, Long SK. Health care spending and service use among highcost Medicaid beneficiaries, 2002-2004. Inquiry. 2009-2010;46(4):405-17.

4. Zulman DM, Pal Chee C, Wagner TH, et al. Multimorbidity and healthcare utilisation among high-cost patients in the US Veterans Affairs Health Care System. BMJ Open. 2015;5(4):e007771.

5. Wammes JJG, van der Wees PJ, Tanke MAC, Westert GP, Jeurissen PPT. Systematic review of high-cost patients' characteristics and healthcare utilisation. BMJ Open. 2018;8(9):e023113.

6. Bommer C, Sagalova V, Heesemann E, et al. Global economic burden of diabetes in adults: projections from 2015 to 2030. Diabetes Care. 2018;41(5):963-70.

7. Yang W, Dall TM, Halder P, et al. Economic costs of diabetes in the US in 2012. Diabetes Care. 2013;36(4):1033-46.

8. Meyers JL, Parasuraman S, Bell KF, Graham JP, Candrilli SD. The highcost, type 2 diabetes mellitus patient: an analysis of managed care administrative data. Arch Public Health. 2014;72(1):6.

9. Sherman RE, Anderson SA, Dal Pan GJ, et al. Real-world evidence - what is it and what can it tell us? N Engl J Med. 2016;375(23):2293-97.

10. U.S. Food and Drug Administration. Framework for FDA's real-world evidence program. December 2018. Available at: https://www.fda.gov/ media/120060/download. Accessed November 12, 2019.

11. Malone DC, Brown M, Hurwitz JT, Peters L, Graff JS. Real-world evidence: useful in the real world of US payer decision making? How? When? And what studies? Value Health. 2018;21(3):326-33.

12. Rassen JA, Solomon DH, Glynn RJ, Schneeweiss S. Simultaneously assessing intended and unintended treatment effects of multiple treatment options: a pragmatic "matrix design." Pharmacoepidemiol Drug Saf. 2011;20(7):675-83.

13. Wang SV, Verpillat P, Rassen JA, Patrick A, Garry EM, Bartels DB. Transparency and reproducibility of observational cohort studies using large healthcare databases. Clin Pharmacol Ther. 2016;99(3):325-32.

14. Kim SC, Solomon DH, Rogers JR, et al. Cardiovascular safety of tocilizumab versus tumor necrosis factor inhibitors in patients with rheumatoid arthritis: a multi-database cohort study. Arthritis Rheumatol. 2017;69(6):1154-64

15. Cramer JA. A systematic review of adherence with medications for diabetes. Diabetes Care. 2004;27(5):1218-24.

16. Polonsky WH, Henry RR. Poor medication adherence in type 2 diabetes: recognizing the scope of the problem and its key contributors. Patient Prefer Adherence. 2016;10:1299-307.

17. Blackburn DF, Swidrovich J, Lemstra M. Nonadherence in type 2 diabetes: practical considerations for interpreting the literature. Patient Prefer Adherence. 2013;7:183-89.

18. Lewey J, Wei W, Lauffenburger JC, et al. Targeted adherence intervention to reach glycemic control with insulin therapy for patients with diabetes (TARGIT-Diabetes): rationale and design of a pragmatic randomised clinical trial. BMJ Open. 2017;7(10):e016551.

19. Lauffenburger JC, Lewey J, Jan S, et al. Rationale and design of the ENhancing outcomes through Goal Assessment and Generating Engagement in Diabetes Mellitus (ENGAGE-DM) pragmatic trial. Contemp Clin Trials. 2017;59:57-63

20. Wammes JJG, van der Wees PJ, Tanke MAC, Westert GP, Jeurissen PPT Systematic review of high-cost patients' characteristics and healthcare utilisation. BMJ Open. 2018;8(9):e023113. 


\section{Actionable Real-World Evidence to Improve Health Outcomes and Reduce Medical Spending Among Risk-Stratified Patients with Diabetes}

21. Niefeld MR, Braunstein JB, Wu AW, Saudek CD, Weller WE, Anderson GF. Preventable hospitalization among elderly Medicare beneficiaries with type 2 diabetes. Diabetes Care. 2003;26(5):1344-49.

22. Choudhry NK, Fischer MA, Avorn J, et al. The implications of therapeutic complexity on adherence to cardiovascular medications. Arch Intern Med. 2011;171(9):814-22.

23. Joynt KE, Gawande AA, Orav EJ, Jha AK. Contribution of preventable acute care spending to total spending for high-cost Medicare patients. JAMA. 2013;309(24):2572-78

24. Vuik SI, Mayer EK, Darzi A. Patient segmentation analysis offers significant benefits for integrated care and support. Health Aff (Millwood). 2016;35(5):769-75

25. Gingery D. US FDA wants more examples of real-world data use. Pink Sheet. August 5, 2018. Available at: https://pink.pharmaintelligence.informa. com/PS123625/US-FDA-Wants-More-Examples-Of-RealWorld-DataUse?vid=Pharma. Accessed November 12, 2019.

26. Shaywitz D. Should tech entrepreneurs aspire to fix clinical trials or reinvent them? Forbes. May 22, 2018. Available at: https://www.forbes.com/sites/ davidshaywitz/2018/05/22/should-tech-entrepreneurs-aspire-to-fix-clinicaltrials-or-reinvent-them/\#5e0blf28775c. Accessed November 12, 2019.

27. Wang SV, Schneeweiss S, Berger ML, et al. Reporting to improve reproducibility and facilitate validity assessment for healthcare database studies V1.0. Pharmacoepidemiol Drug Saf. 2017;26(9):1018-32.

28. von Elm E, Altman DG, Egger M, Pocock SJ, Gøtzsche PC,

Vandenbroucke JP; STROBE Initiative. The Strengthening the Reporting of Observational Studies in Epidemiology (STROBE) statement: guidelines for reporting observational studies. Epidemiology. 2007;18(6):800-04.

29. Benchimol E, Smeeth L, Guttmann A, et al. The REporting of studies Conducted using Observational Routinely-collected health Data (RECORD) statement. PLoS Med. 2015;12(10):e1001885.
30. Zinman B, Wanner C, Lachin JM, et al. Empagliflozin, cardiovascular outcomes, and mortality in type 2 diabetes. N Engl J Med. 2015;373(22):2117-28.

31. Neal B, Perkovic V, Mahaffey KW, et al. Canagliflozin and cardiovascular and renal events in type 2 diabetes. N Engl J Med. 2017;377:644-57.

32. Mahaffey KW, Neal B, Perkovic V, et al.; CANVAS Program Collaborative Group. Canagliflozin for primary and secondary prevention of cardiovascular events: results from the CANVAS program (Canagliflozin Cardiovascular Assessment Study). Circulation. 2018;137(4):323-34.

33. American Diabetes Association. Standards of medical care in diabetes-2019. Diabetes Care. 2019;42(Suppl 1):S1-S187.

34. Franklin JM, Schneeweiss S. When and how can real world data analyses substitute for randomized controlled trials? Clin Pharmacol Ther. 2017;102(6):924-33

35. Patorno E, Goldfine AB, Schneeweiss S, et al. Cardiovascular outcomes associated with canagliflozin versus other non-gliflozin antidiabetic drugs: population based cohort study. BMJ. 2018;360:k119.

36. Patorno E, Pawar A, Franklin JM, et al. Empagliflozin and the risk of heart failure hospitalization in routine clinical care. Circulation. 2019;139(25):2822-30

37. Schneeweiss S, Avorn J. A review of uses of health care utilization databases for epidemiologic research on therapeutics. J Clin Epidemiol. 2005;58(4):323-37.

38. Zelniker TA, Wiviott SD, Raz I, et al. Comparison of the effects of glucagon-like peptide receptor agonists and sodium-glucose co-transporter 2 inhibitors for prevention of major adverse cardiovascular and renal outcomes in type 2 diabetes mellitus: a systematic review and meta-analysis of cardiovascular outcomes trials. Circulation. 2019;139(17):2022-31. 


\section{Actionable Real-World Evidence to Improve Health Outcomes and Reduce Medical Spending Among Risk-Stratified Patients with Diabetes}

\section{APPENDIX A Outcome Definitions}

Measure

Albuminuria

Angina

Heart failure

hospitalization

Hypoglycemia

Kidney failure

hospitalization

Diabetic ketoacidosis

Metabolic failure

Microvascular complications

Myocardial infarction

hospitalization

Percutaneous

revascularization

Stroke

Urinary tract

infections

CPT-4 = Current Procedural Terminology, 4th edition; ER=emergency room, ICD-9/10-CM = International Classification of Diseases, Ninth/Tenth Revision, Clinical

Modification; IP = inpatient.

\section{Measure Definition}

At least 1 of the following: albumin/creatinine value $>300 \mathrm{ug} / \mathrm{mg}$, albumin mass/volume in urine value $>200 \mathrm{mg} / \mathrm{L}$, or albumin mass per volume value $>300 \mathrm{mg} / 24 \mathrm{hr}$

ICD-9/10-CM in any position: 413.x, 414.0x/I20.9, I25.1x

ICD-9/10-CM in primary position: 428.x, 398.91, 402.xl, 404.xl, 404.x3 / I50.x, I09.81, Il1.0, I13.0, I13.2

AND Claim Place of Service Code is any of: ER, IP

ICD-9/10-CM in any position: 249.8, 250.3, 250.8, 251.0, 251.2/E11.64, E13.64, E16.0, E16.1, E16.2

ICD-9/10-CM in any position: 584.x, 586/N17.x, N19

AND Claim Place of Service Code is any of: ER, IP

ICD-9/10-CM in any position: 250.1x/E10.10, E11.69, E13.10 Initiation of insulin as proxy

ICD-9/10-CM in any position: 250.40, 250.42, 250.50, 250.52, 250.60, 250.62, 250.7, 250.70, 250.72, 337.1, 354.x, 355.x, 357.2, 362.x, 365.44, 366.41, 583.81, 713.5/E11.2x, E13.2x, E11.3x, E11.4x, E11.5x, E13.3x, E13.4x, E13.5x, G59, G99.0, M14.6x

ICD-9/10-CM in primary position: 410.x/I21.09, I21.11, I21.19, I21.29, I21.3, I21.4

AND Claim Place of Service Code is any of: ER, IP

CPT-4/ICD-9-CM procedure in any position: 92920, 92921, 92924, 92925, 92937, 92938, 92941, 92943, 92944, 92973, 92984;

$92928,92929,92933,92934,33510,33536,33511,33512,33513,33514,33517,33518,33519,33521,33522,33533,33534$,

$33535 / 00.66,36.03,36.06,36.07,36.09,36.1 x$

OR ICD-9/10-CM in any position: V45.81, 414.04, 414.05/T82.2, Z95.1

AND Claim Place of Service Code is any of: ER, IP

ICD-9/10-CM in any position: 430, 431, 432.x, 433.x, 434.x, 435.x, 436, 437.x, 438.x/I60.x, I61.x, I62.x, I63.x, I65.x, I66.x, G45.x, G46.x, I67.x, I68.2, I68.8

ICD-9/10-CM in any position: 590.0x, 590.1x, 590.8, 590.9, 595.x, 597.8x, 599.0/N11.x, N10, N12, N15.9, N30.x, N34.x, N39.0 
Actionable Real-World Evidence to Improve Health Outcomes and Reduce

Medical Spending Among Risk-Stratified Patients with Diabetes

APPENDIX B Antidiabetic Treatment Utilization Pattern in Members with T2DM

T2DM patients
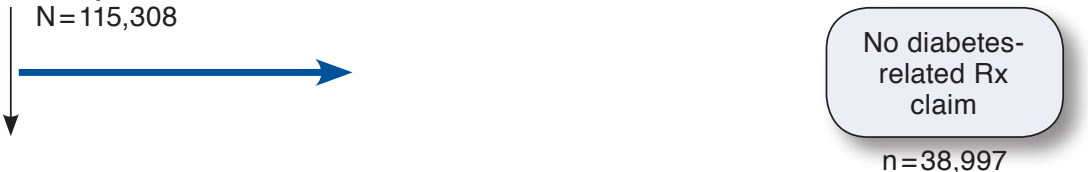

Metformin only
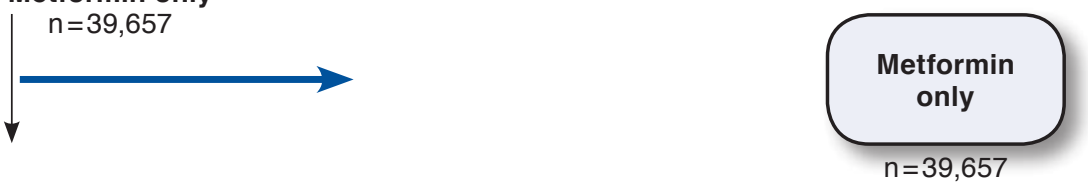

Dual therapy

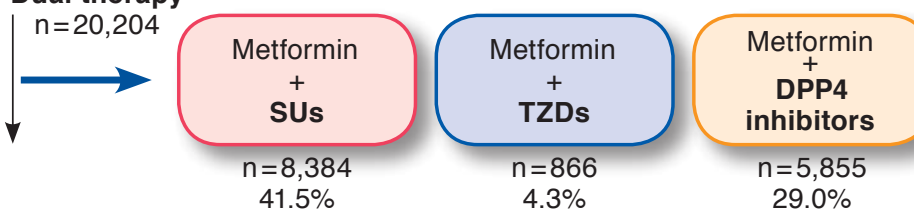

Other combination therapy
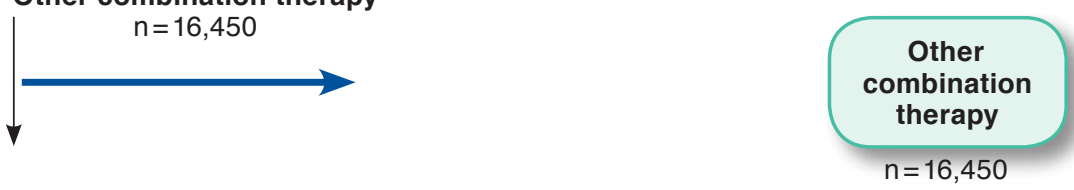

DPP-4 = dipeptidyl peptidase-4; GLP-1 = glucagon-like peptide-1; Rx = prescription; SGLT-2 = sodium-glucose co-transporter-2; SU = sulfonylurea; T2DM = type 2 diabetes mellitus; TZD = thiazolidinedione. 